\title{
Desempenho motor fino e funcionalidade em crianças com síndrome de Down
}

Fine motor performance and functionality in children with Down syndrome

Aline Cirelli Coppede', Ana Carolina de Campos², Denise Castilho Cabrera Santos ${ }^{3}$, Nelci Adriana Cicuto Ferreira Rocha ${ }^{4}$

RESUMO I O objetivo do estudo foi comparar crianças com síndrome de Down (SD) e crianças típicas quanto ao desempenho motor fino, avaliado pela Bayley Scales of Infant and Toddler Development - Third Edition (BSITD-III), e o desempenho em autocuidado segundo o Inventário Pediátrico de Avaliação de Incapacidade (PEDI); e investigar associação entre ambos os domínios. Participaram 12 crianças típicas e 12 crianças com SD, avaliadas na idade de 2 anos. As crianças com SD apresentaram desempenho motor fino e funcionalidade inferior às crianças típicas, possivelmente por dificuldades em desempenhar tarefas que exijam destreza e coordenação manual, como as que compõem a BSITD-III. Apesar disso, sua pontuação em autocuidado foi adequada para a faixa etária, possivelmente porque as habilidades funcionais exigidas nesse período, como retirar calçados/vestimenta, impõem menor demanda motora fina do que tarefas da BSITD-III. Esse fato pode ter contribuído para o bom desempenho funcional das crianças com SD, e para a ausência de associação entre os domínios. Fatores como os cuidados oferecidos à criança pelos cuidadores, bem como ambientes estimuladores provavelmente também contribuíram para os resultados.

Descritores I desenvolvimento infantil; destreza motora; atividades cotidianas; síndrome de Down.
ABSTRACT I The aim of study was to compare typicallydeveloping children and children with Down syndrome (DS) for their fine motor performance, measured by the Bayley Scales of Infant and Toddler Development - Third Edition (BSITD-III), and for their functional performance in self-care, measured by the Pediatric Evaluation of Disability Inventory (PEDI). Associations between these areas were also investigated. Twelve typically-developing children and 12 children with DS were assessed at the age of 2 years. The children with DS performed poorly in fine motor skills when compared with typical children, which may be explained by the complex motor skills involved in the BSITDIll's tasks. Their self-care scores were also lower in comparison with the typical children. However, these scores fell under the average performance for children without disabilities, which may be explained by the low complexity, in terms of fine motor skills, of functional tasks expected for children at that age (eg.: taking shoes/pants off). This finding has probably contributed to the lack of correlation between the areas. Factors such as the assistance provided by the caregivers, and environmental stimulation, may also have contributed to the results.

Keywords I child development; motor skills; activities of daily living; Down syndrome.

\footnotetext{
Estudo desenvolvido no Curso de Especialização em Intervenção em Neuropediatria do Centro de Ciências Biológicas e da Saúde da Universidade Federal de São Carlos (UFSCar) - São Carlos (SP) Brasil.

'Doutoranda em Educação Especial na UFSCar - São Carlos (SP) Brasil;

${ }^{2}$ Doutoranda em Fisioterapia na UFSCar - São Carlos (SP) Brasil;

${ }^{3}$ Coordenadora do Curso de Mestrado em Fisioterapia da Faculdade de Ciências da Saúde da Universidade Metodista de Piracicaba -

FACIS/UNIMEP - Piracicaba (SP), Brasil.

${ }^{4}$ Professora Adjunta do Curso de Fisioterapia da UFSCar - São Carlos (SP) Brasil.
} 


\section{INTRODUÇÃO}

As habilidades de alcançar, apreender e manipular objetos são essenciais para o desenvolvimento infantil, emergindo a partir dos quatro meses de vida ${ }^{1}$. Ao completar dois anos de idade, crianças com desenvolvimento típico são capazes de realizar ações manuais maduras e funcionais ${ }^{2}$, demonstrando planejamento antecipado dos movimentos, uso coordenado das duas mãos e seleção de padrões de apreensão adequados às características dos objetos durante a realização de atividades motoras finas ${ }^{3}$.

Fatores ambientais influenciam diretamente o desenvolvimento motor nos primeiros anos $\mathrm{de} \mathrm{vida}^{4}$, sendo observado que ambientes ricos em estímulos favorecem o desenvolvimento motor, com destaque para habilidades motoras finas ${ }^{5,6,7}$, e por outro lado, crianças criadas em ambientes superprotetores podem ser menos independentes ${ }^{8,9}$. Além disso, fatores de risco como nascimento pré-termo, síndromes e outras condições podem interferir no desenvolvimento da motricidade fina ${ }^{10}$, possivelmente afetando a participação das crianças em tarefas como vestir-se, alimentar-se e brincar ${ }^{11}$.

No que se refere à síndrome de Down (SD), restrições intrínsecas como a hipotonia muscular e a hipermobilidade articular contribuem para o atraso no desenvolvimento motor, para a lentidão na realização dos movimentos e para alterações no controle postural ${ }^{12-14}$. Essas características diminuem a possibilidade de experiências motoras e de exploração do ambiente ${ }^{15}$, o que prejudica o desempenho das habilidades motoras finas e destreza manual ${ }^{8,15-18}$.

Estudos sugerem que crianças com $\mathrm{SD}$ aos dois e cinco anos apresentam baixo desempenho funcional nas áreas de mobilidade, autocuidado e função social ${ }^{8,17,19,20}$. Estudos com crianças com SD em idade escolar demonstram que dificuldades motoras interferem no desempenho de atividades de vida diária, dentre elas as tarefas de autocuidado ${ }^{11-14,18,19}$. No entanto, em crianças mais jovens tal relação não tem sido investigada.

Diante do exposto, o objetivo do estudo foi comparar o desempenho motor fino e o desempenho de habilidades funcionais de autocuidado de crianças com SD e crianças típicas na faixa etária de dois anos, e verificar se existe relação entre esses domínios.

Tendo em vista as características neuromotoras de crianças com $\mathrm{SD}^{8,17,18,20}$, esperou-se que o perfil motor fino e funcional nas habilidades de autocuidado fossem atrasados em comparação com crianças típicas. Ainda, considerando a importância das habilidades motoras finas para o desempenho de tarefas de autocuidado, levantou-se a hipótese de que dificuldades motoras sejam relacionadas ao baixo desempenho funcional. Os dados obtidos auxiliariam na caracterização de limitações funcionais em crianças com SD e subsidiariam estratégias de intervenção especializada e adaptativa.

\section{MÉTODOS}

O presente estudo tem desenho transversal do tipo caso-controle. Participaram 24 crianças de ambos os gêneros, nascidas a termo, e com peso adequado para idade gestacional, sendo 12 crianças pertencentes ao grupo típico $(\mathrm{T})$, com idade média de 26,71 meses $( \pm 1,84)$; e 12 crianças com síndrome de Down (SD) com idade média de 27,15 meses $( \pm 2,04)$.

As crianças com SD foram recrutadas em duas instituições que forneciam atendimento de estimulação precoce em duas cidades do interior paulista de médio porte. As crianças do grupo típico foram recrutadas em escolas de educação infantil. Foram considerados critérios de inclusão crianças com idade gestacional de 37 a 41 semanas e 7 dias, peso adequado para idade gestacional, e com idade mínima de 24 meses e máxima de 31 meses, que tinham capacidade de compreender comandos simples. No grupo típico, os critérios incluíam ausência de alterações neurológicas, motoras e sensoriais, e no grupo SD, diagnóstico de trissomia do cromossomo 21 e participação de programas de intervenção motora.

O estudo foi aprovado pelo Comitê de Ética e Pesquisa local (Protocolo no 228/2009). Antes da avaliação, os responsáveis assinaram o termo de consentimento livre e esclarecido e responderam a um questionário sobre o desenvolvimento da criança e critério de classificação socioeconômica.

Como instrumento de avaliação do desempenho motor fino foi utilizada a Bayley Scales of Infant and Toddler Development-BSITD-III ${ }^{21}$,e como instrumento de avaliação de habilidades funcionais foi utilizado o Pediatric Evaluation of Disability Inventory (PEDI) ${ }^{22}$.

Neste estudo utilizou-se a subescala motora fina da BSITD-III, composta de 66 tarefas. Essa escala pode ser aplicada em crianças de 1 até 42 meses, e as tarefas avaliadas são pontuadas como 1 , quando a criança realiza a tarefa conforme descrito no manual, e 0 quando não realiza, ou realiza de forma inadequada.

As tarefas avaliadas considerando a faixa etária dos participantes foram: empilhamento de blocos, imitar 
traços, colocar cereal no pote, preensão do giz ou lápis, colocar moedas no cofre, encaixar e desencaixar blocos, passar o cadarço pelo orifício dos blocos, imitar movimentos das mãos, picotar papel com tesoura, discriminar formas pelo tato, abotoar, imitar desenhos (sinal de mais e quadrado) e recortar respeitando os limites da linha. $\mathrm{O}$ conjunto de tarefas avaliado não é necessariamente o mesmo para todas as crianças, havendo critérios de idade e desempenho mínimo estabelecido pelo instrumento para determinação do início e fim da avaliação. Ao final da avaliação o desempenho da criança é expresso por um escore (scaled score) que varia de 1 a 19 pontos, com média 10 e desvio padrão 3 , representando o desempenho da criança em relação à média normativa da escala. Escores dentro do intervalo de 7 a 13 pontos são considerados dentro dos limites normais. Escores entre 4 e 7 pontos são classificados como comprometimento leve; escores de 3 a 1 ponto são classificados como comprometimento moderado; e escore 0 , grave.

Com relação ao PEDI, neste estudo foi utilizado o questionário de autocuidado da parte I, que documenta o desempenho da criança nas áreas de alimentação, higiene pessoal, banho, vestir, uso do toalete e controle esfincteriano. Esse questionário pode ser aplicado em crianças de seis meses a sete anos e seis meses de idade. A pontuação da criança é obtida através de um Escore Normativo, que vai de 0 a 100 , com média 50 $(\mathrm{DP}=20)$, informando o desempenho da criança em relação ao desempenho esperado de crianças típicas da mesma faixa etária.

As avaliações foram realizadas por duas pessoas treinadas e após obtenção do índice de concordância interobservador de $91 \%$.

\section{Análise estatística}

Após verificada a normalidade da amostra por meio do teste de Shapiro-Wilk, empregou-se o teste $t$ para comparar os grupos quanto ao scaled score na BSITDIII e escore normativo no PEDI.

O teste de Fisher comparou a frequência de crianças que pontuaram nas tarefas da Bayley. As tarefas selecionadas pertencem às séries empilhar blocos (T31, T38 e T54), imitar traços (T32, T40, T41 e T43) e preensão (T28, T34, T37 e T48).

O teste de correlação de Pearson investigou relação entre as pontuações obtidas por meio da BSITD-III e as pontuações do $\mathrm{PEDI}$.

Para todas as análises foi considerado um nível de significância de 5\%.
Tabela 1. Caracterização das famílias dos participantes quanto ao nível de escolaridade, nível socioeconômico e quantidade de filhos

\begin{tabular}{lccc} 
& & Grupo & Grupo \\
\cline { 3 - 4 } & & SD & T \\
Nivel de Escolaridade & SC & $50 \%$ & $60 \%$ \\
Materna & MC & $25 \%$ & $20 \%$ \\
& FC & ---- & $20 \%$ \\
ABIPEME & FI & $25 \%$ & - \\
& B & $37,5 \%$ & $40 \%$ \\
& C & $50 \%$ & $60 \%$ \\
No de Filhos & D & $12,5 \%$ & - \\
& 1 & $37,5 \%$ & $87,5 \%$ \\
& 2 & $37,5 \%$ & $12,5 \%$ \\
& 3 & $25 \%$ & $12,5 \%$ \\
& 5 & --- & $12,5 \%$
\end{tabular}

SC: superior completo; MC: Ensino Médio completo; FC: Ensino Fundamental completo; FI: fundamental incompleto; ABIPEME: Critério de classificaçăo socioeconômica Brasil (2008)

\section{RESULTADOS}

O grupo Síndrome de Down (SD) apresentou pontuação significativamente menor $(t(22)=9,14$; $\mathrm{p}<0,0001)$ no scaled score segundo a BSITD-III, com média de 4,67 $( \pm 1,5)$, enquanto o grupo típico $(\mathrm{T})$ apresentou média de 13,14 $( \pm 2,1)$. Considerando que o scaled score tem média $10( \pm 3)$, no grupo SD dois participantes obtiveram pontuação entre 10 e 7 nove participantes obtiveram pontuação entre 7 e 4 , indicando atraso leve no desenvolvimento, e uma criança teve pontuação 3, indicatndo atraso moderado.

No grupo T, três participantes obtiveram pontuação acima de 13 e os outros nove participantes obtiveram pontuação entre 13 e 9 , ou seja, dentro dos limites de normalidade.

Quanto ao PEDI, o grupo SD apresentou pontuação significativamente menor $(t(11)=5,426 ; p<0,0001)$ no Escore Normativo $(\mathrm{M}=33,8 \pm 8,0)$ quando comparado com o grupo $\mathrm{T}(\mathrm{M}=56,2 \pm 6,9)$.

Considerando que o Escore Normativo tem média 50 ( \pm 20 ), no grupo SD, 11 participantes obtiveram pontuação entre 50 e 30 (dentro do intervalo de normalidade), sendo a maior pontuação 43,7. Apenas um sujeito obteve pontuação 20,3 , ou seja, abaixo do intervalo de normalidade.

No grupo $\mathrm{T}$ todos os indivíduos obtiveram pontuação dentro do intervalo de normalidade, sendo a menor pontuação de valor 50,3 e a maior de valor 68 .

Com relação à frequência das respostas e a análise comparativa entre os grupos para as provas da série empilhar blocos da BSITD-III, a Tabela 2 mostra que houve diferença significativa entre os grupos na tarefa T38 (empilhar até seis blocos). Na tarefa T54, nenhuma 
criança do grupo SD chegou a ser avaliada neste item durante o teste.

A Tabela 3 mostra que para a série imitar traços houve diferença significativa entre grupos apenas na tarefa T41 (fazer traço na vertical), em que nenhuma das crianças do grupo SD pontuou.

$\mathrm{Na}$ série preensão houve diferença significativa entre os grupos na tarefa T37 (preensão intermediária - tripé estático), não realizada pela maior parte das crianças do grupo SD. Somente uma criança do grupo SD chegou até a tarefa T48 e não pontuou nesse item. No grupo T, todas as crianças chegaram até esse item; porém, também não pontuaram (Tabela 4).

Em todas as tarefas o grupo $\mathrm{SD}$, comparado ao grupo $\mathrm{T}$, manteve-se com menor frequência de respostas executadas.

Não houve correlação significativa entre as pontuaçãos obtidas na BSITD-III e no PEDI, sendo no grupo $\mathrm{SD}(\mathrm{r}=0,40 ; \mathrm{p}=0,43)$ e no grupo $\mathrm{T}(\mathrm{r}=0,29 ; \mathrm{p}=0,53)$.

\section{DISCUSSÃO}

Confirmando a hipótese previamente levantada, as crianças com SD apresentaram desempenho motor fino inferior ao grupo típico, a maioria com atraso leve. É esperado que ao final do primeiro ano de idade comece a se desenvolver a preensão de precisão, que requer o controle da musculatura intrínseca da mão e movimentos independentes dos dedos, necessários para manipular objetos pequenos e delicados ${ }^{2,3,8}$. Os resultados do presente estudo mostram que já nessa fase as crianças com SD apresentam dificuldades na execução de tarefas motoras finas.

A análise de séries individuais revelou aspectos mais detalhados sobre o desempenho dos participantes.

O grupo com SD teve desempenho inferior na tarefa de empilhar até seis ou mais blocos (T38 e T54) e fazer traço na vertical (T41). Tais atividades, presentes no cotidiano de crianças na faixa etária estudada, envolvem coordenação olho-mão e controle dos dedos, ajudando a estabelecer as bases para habilidades motoras finas complexas exigidas em idades posteriores, como escrita e manipulação de pequenos objetos. Contribuem, ainda, para desenvolver conceitos de agrupamento, causa e efeito, relação entre objetos e solução de problemas $^{2,16}$. Portanto, é possível que as dificuldades apresentadas por crianças com $\mathrm{SD}$ em idade escolar sejam decorrentes de limitações presentes em fases anteriores do desenvolvimento.

$\mathrm{Na}$ série preensão, os grupos diferiram apenas na tarefa T37 (preensão intermediária - tripé estático), sendo que a maioria das crianças do grupo SD executam preensão palmar e transicional, e a maioria das crianças do grupo $\mathrm{T}$ executam preensão transicional e intermediária. Nenhuma criança do grupo $T$ apresentou preensão palmar em seu repertório, ou seja, apresentaram formas mais elaboradas de preensão, enquanto a maior parte das crianças com SD realizou essa estratégia. Esses resultados

Tabela 2. Distribuição da frequência de respostas entre os grupos T e SD para as provas da série empilhar blocos.

\begin{tabular}{lccccc}
\multirow{2}{*}{ Tarefas } & Grupo & $\mathrm{n}$ & Não executa & Executa & \\
\cline { 5 - 5 } & & & $f(\%)$ & $f(\%)$ & \\
T31 & $\mathrm{T}$ & 12 & $1(8,33)$ & $11(91,66)$ & 0,06 \\
& $\mathrm{SD}$ & 12 & $6(50,0)$ & $6(50,0)$ & \\
T38 & $\mathrm{T}$ & 12 & $2(16,6)$ & $10(83,33)$ & $<0,0001$ \\
& $\mathrm{SD}^{*}$ & 10 & $10(100,0)$ & $\mathrm{O}(0)$ & \\
T54 & $\mathrm{T}^{*}$ & $\mathrm{O}$ & $3(50,0)$ & $3(50,0)$ & \\
& $\mathrm{SD}^{* *}$ & $\mathrm{O}$ & $\mathrm{O}(0)$ & $\mathrm{O}(0,0)$ &
\end{tabular}

T31: empilhar até 2 blocos; T38: empilhar até 6 blocos; T54: empilhar até 8 blocos; T: típico; SD: Sindrome de Down; f: frequência observada; *2 SD e 6 T não chegaram nessa tarefa durante a avaliação: **nenhum SD chegou nessa tarefa durante a avaliação

Tabela 3. Distribuição da frequência de respostas entre os grupos T e SD para as provas da série imitar traços

\begin{tabular}{|c|c|c|c|c|c|}
\hline \multirow{2}{*}{ Tarefas } & \multirow{2}{*}{ Grupo } & \multirow{2}{*}{$n$} & Não executa & Executa & \multirow{2}{*}{ Valor $p$} \\
\hline & & & $f(\%)$ & $f(\%)$ & \\
\hline \multirow{2}{*}{ T32 } & $\mathrm{T}$ & 12 & $\mathrm{O}(0,0)$ & $12(100,0)$ & \multirow[t]{2}{*}{0,09} \\
\hline & SD & 12 & $4(33,3)$ & $8(66,7)$ & \\
\hline \multirow{2}{*}{ T40 } & $T$ & 12 & $7(58,33)$ & $5(41,66)$ & \multirow[t]{2}{*}{0,32} \\
\hline & $\mathrm{SD}^{*}$ & 8 & $7(87,5)$ & $1(12,5)$ & \\
\hline \multirow{2}{*}{ T41 } & $\mathrm{T}$ & 12 & $6(50,0)$ & $6(50,0)$ & \multirow[t]{2}{*}{0,04} \\
\hline & $S D^{* *}$ & 7 & $7(100,0)$ & $\mathrm{O}(0,0)$ & \\
\hline \multirow{2}{*}{ T43 } & $T$ & 12 & $4(33,3)$ & $8(66,7)$ & \multirow[t]{2}{*}{0,52} \\
\hline & $S D^{* \star *}$ & 3 & $2(66,7)$ & $1(33,3)$ & \\
\hline
\end{tabular}

T32: traço em alguma direção; T40: traço horizontal; T41: traço vertical; T43: traço circular; T: típico; SD: Síndrome de Down; sujeitos; f: frequência observada; *4 SD não chegaram nessa tarefa durante a avaliação; ** 5 SD não chegaram nessa tarefa durante a avaliação; ***9 SD não chegaram nessa tarefa durante a avaliação

Tabela 4. Distribuição da frequência de respostas entre os grupos T e SD para as provas da série preensão

\begin{tabular}{|c|c|c|c|c|c|}
\hline \multirow{2}{*}{ Tarefas } & \multirow{2}{*}{ Grupo } & \multirow{2}{*}{$\mathrm{n}$} & Não executa & Executa & \multirow{2}{*}{ Valor $p$} \\
\hline & & & $f(\%)$ & $f(\%)$ & \\
\hline \multirow{2}{*}{$\mathrm{T} 28$} & $\mathrm{~T}$ & 12 & $\mathrm{O}(0, \mathrm{O})$ & $12(100,0)$ & \multirow[t]{2}{*}{1,0} \\
\hline & SD & 12 & $1(8,3)$ & $11(91,7)$ & \\
\hline \multirow{2}{*}{ T34 } & $\mathrm{T}$ & 12 & $1(8,3)$ & $11(91,7)$ & \multirow[t]{2}{*}{0,32} \\
\hline & SD & 12 & $4(33,3)$ & $8(66,7)$ & \\
\hline \multirow{2}{*}{ T37 } & $\mathrm{T}$ & 12 & $3(25,0)$ & $9(75,0)$ & \multirow[t]{2}{*}{0,039} \\
\hline & $S D^{*}$ & 11 & $8(72,7)$ & $3(27,3)$ & \\
\hline \multirow{2}{*}{ T48 } & $T$ & 12 & $12(100,0)$ & $\mathrm{O}(0,0)$ & \\
\hline & $S D^{* *}$ & 1 & $1(100,0)$ & $\mathrm{O}(0,0)$ & \\
\hline
\end{tabular}

T28: preensão palmar; T34: preensão transicional; T37: preensão intermediária; T48: preensão dinâmica; T: típico: SD: Síndrome de Down; f: frequência observada: *1 SD não chegou nessa tarefa durante a avaliação; **11 SD não chegaram nessa tarefa durante a avaliação 
confirmam os resultados de Jover et al. ${ }^{15}$, de padrões de preensão imaturos em crianças com SD. Somente uma criança do grupo SD chegou até o item T48 e não pontuou na tarefa; no grupo $\mathrm{T}$, todas as crianças chegaram até esse item, porém, também não pontuaram.

Tais resultados permitem inferir que habilidades que envolvem controle e planejamento motor, percepção visual e integração visomotora ainda precisam ser amadurecidas nas crianças com síndrome de Down. Dificuldades em ativar/controlar a musculatura intrínseca da mão podem ter contribuído para os achados, além de fatores como a adoção de padrões atípicos de preensão - por exemplo, usar menos dedos, e realizar excessiva extensão dos dedos ao agarrar, relatados em crianças com $\mathrm{SD}^{15}$.Por outro lado, crianças típicas na faixa etária de dois anos ainda não apresentam a motricidade fina desenvolvida por completo ${ }^{18,19}$.

Quanto às habilidades funcionais de autocuidado, as crianças com $\mathrm{SD}$ obtiveram pontuação inferior às típicas, confirmando as hipóteses levantadas. Esses resultados são semelhantes a estudos prévios, que têm atribuído tal atraso às características da síndrome e também à superproteção dos pais, que pode limitar a participação das crianças nas atividades de autocuidado ${ }^{9,20}$.

No entanto, diferentemente do observado em outros estudos, a maioria das crianças com SD avaliada no presente estudo obteve pontuação dentro do intervalo de normalidade, ou seja, suas habilidades de autocuidado podem estar dentro do esperado para a faixa etária. Tendo em vista que todas as crianças com SD que participaram deste estudo estavam inseridas em programas de estimulação motora, fatores ambientais podem ter promovido melhor desempenho nas tarefas exigidas no PEDI. De fato, estudos referem que avanços tecnológicos e científicos na área da reabilitação e novas técnicas de estimulação do desenvolvimento infantil contribuem para que o desenvolvimento na SD aproxime-se do desenvolvimento típico ${ }^{9,23}$.

As tarefas que apresentaram maiores dificuldades para ambos os grupos, mas principalmente para as crianças com SD, foram as relacionadas a higiene oral, atividades de vestir e despir utilizando fechos, zíper ou botões, calçar sapatos e o uso do toalete. Uma possível explicação para as "dificuldades" apresentadas por ambos os grupos é que essas limitações sejam próprias da faixa etária analisada, do nível de desenvolvimento motor e cognitivo, e não necessariamente uma limitação funcional. Assim, o fato de ambos os grupos terem apresentado dificuldades em tarefas como manejar fechos e zíperes, assim como usar o toalete independentemente, pode ser atribuído à complexidade destas para as crianças avaliadas.

Em contrapartida, não foi observada correlação entre BSITD-III e PEDI, contrariando a hipótese do estudo e relatos da literatura, que associam o baixo desempenho nas habilidades de autocuidado com a dificuldade nas habilidades motoras finas ${ }^{9,19,21-23}$. Uma possivel explicação para esses resultados é que o desempenho motor fino observado nas crianças com SD resulte de dificuldades em desempenhar tarefas que exijam destreza e coordenação uni e bimanual, como as que compõem a BSITD-III. As habilidades funcionais exigidas para essa faixa etária no PEDI, como retirar calçados/vestimenta, são menos complexas em termos de exigência motora fina do que tarefas da BSITD-III. Esse fato pode ter contribuído para o desempenho funcional dentro do intervalo de normalidade das crianças com SD, e para a ausência de associação entre os domínios. Tendo em vista que em diferentes fases do desenvolvimento as estruturas corporais e habilidades cognitivas interagem diferentemente com as tarefas propostas, mediante experiências vividas, futuros estudos devem avaliar crianças em outras faixas etárias para compreender melhor a relação entre os domínios investigados no presente estudo.

\section{CONCLUSÃO}

As crianças com SD apresentaram perfil motor fino e funcional inferiores às crianças com desenvolvimento típico; porém, o desempenho funcional do grupo SD esteve adequado segundo o esperado para a faixa etária. Apesar de as tarefas de autocuidado exigirem bom desempenho nas habilidades motoras finas, não foi observada relação entre os domínios nessa faixa etária estudada. Os resultados apontam dificuldades específicas em tarefas motoras finas mais complexas. Este achado indica necessidade de intervenção direcionada a esse aspecto, visto que pode influenciar a aquisição de habilidades funcionais posteriormente no desenvolvimento.

\section{REFERÊNCIAS}

1. Rocha NACF, Silva FPS, Tudella E. Influência do tamanho e da rigidez dos objetos nos ajustes proximais e distais do alcance de lactentes. Rev Bras Fisioter. 2006;10(3):263-9. 
2. Geerts WK, Einspieler C, Dibiasi J, Garzarolli B, Bos AF. Development of manipulative hand movements during the second year of life. Early Hum Dev. 2003;75(1-2):91-103.

3. Wuang YP, Wang CC, Huang MH, Su CY. Profiles and cognitive predictors of motor functions among early school-age children with mild intellectual disabilities. J Intellec Disabil Res. 2008;52(12):1048-60.

4. Miquelote AF, Santos DC, Caçola PM, Montebelo MI, Gabbard C. Effect of the home environment on motor and cognitive behavior of infants. Infant Behav Dev. 2012;35(3):329-34.

5. Howley SA, Prasad, SE, Pender NP, Murphy KC. Relationship between reaction time, fine motor control, and visual-spatial perception on vigilance and visual-motor tasks in 22q11.2 Deletion Syndrome. Res Dev Disabil. 2012;33(5):1495-502.

6. Fagard J, Spelke E, Von Hofsten C. Reaching and grasping a moving object in 6-, 8-, and 10-month-old infants: laterality and performance. Inf Behav Dev. 2009;32(2):137-46.

7. Abbott A, Bartlett D, Fanning J, Kramer J. Infant motor development and aspects of the home environment. Pediatric Physical Therapy. 2000:12(2):62-7.

8. Mancini MC, Carvalho e Silva P, Gonçalves SC, Martins Sde M. Comparison of functional performance among children with Down syndrome and children with age-appropriate development at 2 and 5 years of age. Arq Neuropsiquiatr. 2003;61(2B):409-15.

9. Pazin AC, Martins MRI. Desempenho funcional de crianças com Sindrome de Down e a qualidade de vida de seus cuidadores. Rev Neuroc. 2007:15(4):297-303.

10. Bart O, Jarus T, Erez Y, Rosenberg, L. How do young children with DCD participate and enjoy daily activities? Res Dev Disabil. 2011; 32(4):1317-22.

11. King G, Law M, Hurley P, Petrenchik T, Schwellnus H. A developmental comparison of the out-of-school recreation and leisure activity participation of boys and girls with and without physical disabilities. Int J Disabil Dev Educ. 2010;57:77-107.

12. Anson JG, Mawston GA. Patterns of muscle activation in simple reaction-time tasks. Perceptual-motor behavior in Down syndrome. New Zealand: Human Kinetics; 2000. p. 4-24.

13. Shumway-Cook A, Woollacott MH. Dynamics of postural control in the child with Down syndrome. Phys Ther. 1985;65(9):1315-22.

14. Volman MJ, Visser JJ, Lensvelt-Mulders GJ. Functional status in 5 to 7-year-old children with Down syndrome in relation to motor ability and performance mental ability. Disabil Rehabil. 2007:29(1):25-31.

15. Jover M, Ayoun C, Berton C, Carlier M. Specific grasp characteristics of children with trisomy 21. Dev Psychobiol. 2010;52(8):782-93.

16. Zatsiorsky VM, Latash ML. Multifinger prehension: an overview. J Mot Behav. 2008;40(5):446-76.

17. Dolva AS, Coster W, Lilja M. Functional performance in children with Down syndrome. Am J Occup Ther. 2004;58(6):621-9.

18. Leonard S, Msall M, Bower C, Tremont M, Leonard H. Functional status of school-aged children with Down syndrome. J Paediatr Child Health. 2002;38(2):160-5.

19. Jobling A, Mon-Williams M. Motor development in Down syndrome: a longitudinal perspective. Perceptual-motor behavior in Down syndrome. New Zealand: Human Kinetics; 2000. p. 226-47.

20. Silva NL, Dessen MA. Crianças com síndrome de Down e suas interações familiares. Psicol Reflex Crit. 2003;16(3):503-14.

21. Bayley N. Bayley Scales of Infant and Toddler Development III - Motor Scale Kit. San Antonio: Harcourt Assessment; 2005.

22. Mancini MC. Inventário de avaliação pediátrica de incapacidade (PEDI): manual da versão brasileira adaptada. Belo Horizonte: UFMG; 2005

23. Polastri PF, Barela JA. Percepção-ação no desenvolvimento motor de crianças portadoras de síndrome de Down. Revista Sobama. 2002;7(1):1-8. 